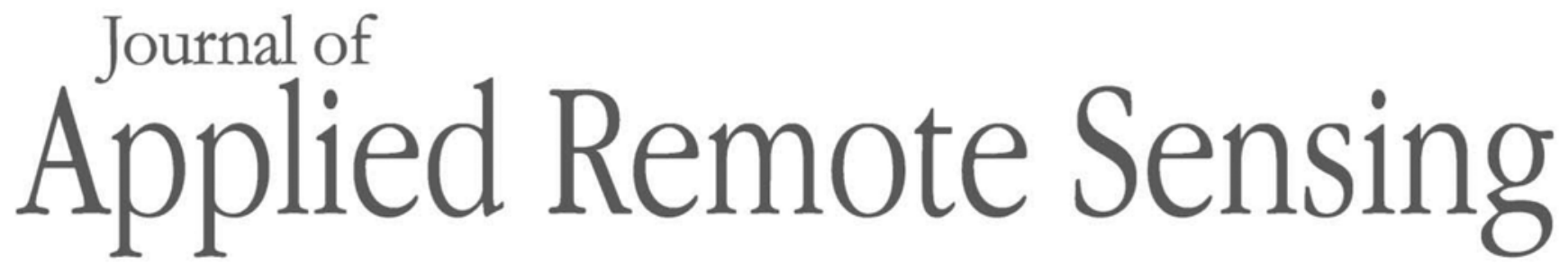

RemoteSensing.SPIEDigitalLibrary.org

\title{
Special Section Guest Editorial: CubeSats and NanoSats for Remote Sensing
}

\author{
Thomas Pagano \\ Charles Norton
}




\title{
Special Section Guest Editorial: CubeSats and NanoSats for Remote Sensing
}

\author{
Thomas Pagano ${ }^{a}$ and Charles Norton ${ }^{b}$ \\ aJet Propulsion Laboratory, California Institute of Technology, Pasadena, \\ California, United States \\ ${ }^{b}$ NASA Headquarters, Science Mission Directorate, Washington, DC, United States
}

There is revolution underway enabled by new advances in instrument technologies, spacecraft design, software, and analysis capabilities enabled by CubeSats and NanoSats for remote sensing. Miniaturization of critical subsystems that can maintain, and in some cases exceed, the capability of legacy systems is influencing the scientific community to reassess what measurements are possible at a fundamental level. That same community has also been inspired to explore how constellation observations can produce data to improve model and forecasting accuracy, and how advances in mathematics can impact the quality of attitude and determination control systems for constellations through platform-independent software systems. They have developed innovative deployable systems for first-of-a-kind remote sensing measurements from CubeSats and new electronics and instrument systems expanding the range of electromagnetic spectrum measurements capable from nanosatellites. They are even exploring how remote sensing can go beyond science to develop instruments and platforms to assess and characterize the performance of space vehicles in flight.

In this special section we have compiled a suite of high-quality papers that run the gamut from fundamentally new measurements, with high-quality science results performed from CubeSat and NanoSat systems, to the development of new remote sensing instruments, to the assessment of the quality of data products that can be expected from CubeSats and their impact on weather prediction. The variety and depth of papers presented give a glimpse into the broad activities on-going in the remote sensing community to expand the reach of CubeSats and NanoSats to play a disruptive role in advancing technologies and measurement approaches for future observations. We thank the authors and the reviewers for their dedication and thoughtfulness in producing this special section.

Thomas Pagano is a systems architect and project manager at NASA's Jet Propulsion Laboratory in Pasadena, California. For the last 17 years, he has managed the Atmospheric Infrared Sounder Project (AIRS), which operates the AIRS and two microwave sounding instruments on the EOS-Aqua Spacecraft. He is the principal investigator for the CubeSat Infrared Atmospheric Sounder (CIRAS) technology development project. He also manages and participates in concept development studies for next-generation imagers and sounders for NOAA. Prior to joining JPL in 1997, he was the chief systems engineer on the MODIS instrument development program at Raytheon SBRS since 1985 and saw this project from concept development through delivery to the spacecraft. He has a BS in physics from UC Santa Barbara and an MS in physics from Montana State University. He holds two US patents on constant spatial resolution imaging techniques. He is a Fellow of SPIE.

Charles D. Norton is the special advisor for small spacecraft missions at NASA HQ. In that role he is responsible for advising on NASA's cross-agency strategic direction for innovative small satellite science, exploration, and technology missions from ESPA-class spacecraft down to CubeSats. Previously, he was a principal technologist and program manager associate at the Jet Propulsion Laboratory, California Institute of Technology, where he developed programs and managed multiple small spacecraft missions for NASA. Charles is a recipient of numerous awards for new technology and innovation, including the JPL Lew Allen Award, Voyager Award, and the NASA Exceptional Service Medal. He has a BSE in electrical engineering and computer science from Princeton University and an MS and PhD in computer science from Rensselaer Polytechnic Institute.

(C) 2019 Society of Photo-Optical Instrumentation Engineers (SPIE) 